\title{
Three-dimensional migration of neutrophils through an electrospun nanofibrous membrane
}

\author{
Songwan Jin ${ }^{1}$, Tae-Min Park², Cho-Hee Kim³ ${ }^{3}$ Jin-Soo Kim ${ }^{1}$, Binh Duong Le', Young Hun Jeong ${ }^{4}$, \\ Jong-Young Kwak ${ }^{5}$, and Sik Yoon ${ }^{6}$ \\ ${ }^{1}$ Department of Mechanical Engineering, Korea Polytechnic University, Siheung, Gyeonggy-do, South \\ Korea, ${ }^{2}$ Department of Advanced Convergence Technology, Korea Polytechnic University, Siheung, \\ Gyeonggy-do, South Korea, ${ }^{3}$ Department of Biochemistry, Dong-A University, Busan, South Korea, \\ ${ }^{4}$ School of Mechanical Engineering, Kyungpook National University, Daegu, South Korea, ${ }^{5}$ Department of \\ Pharmacology, Ajou University School of Medicine, Suwon, Gyeonggy-do, South Korea, and ${ }^{6}$ Department \\ of Anatomy, Pusan National University School of Medicine, Yangsan, Gyeongsangnam-do, South Korea.
}

BioTechniques 58:285-292 (June 2015) doi 10.2144/000114297

Keywords: 3-D cell migration; nanofibrous membrane; electrospinning; extracellular matrix; neutrophil

Supplementary material for this article is available at www.BioTechniques.com/article/114297.

The study of immune cell migration is important for understanding the immune system network, which is associated with the response to foreign cells. Neutrophils act against foreign cells before any other immune cell, and they must be able to change shape and squeeze through narrow spaces in the extracellular matrix (ECM) during migration to sites of infection. Conventional in vitro migration assays are typically performed on two-dimensional substrates that fail to reproduce the three-dimensional (3-D) nature of the ECM. Here we present an in vitro method to simulate the 3-D migration of neutrophils using an electrospun nanofibrous membrane, which is similar to the ECM in terms of morphology. We examined the properties of neutrophil movement and the effects of gravity and the presence of IL-8, which has been widely used as a chemotactic attractant for neutrophils. The number of neutrophils passing through the nanofibrous membrane were higher, and their movement was more active in the presence of IL-8. Also, we confirmed that neutrophils could migrate against gravity toward IL-8 through a nanofibrous membrane.

Cell migration is essential to various biological processes, including wound healing, angiogenesis, cancer metastasis, and immune responses. In particular, the migration of immune cells out of the circulatory system toward damaged or inflamed tissue is a vital step in both innate and adaptive immune responses. Neutrophils are the first responders of the immune system, migrating to sites of infection before any other cells. Neutrophil migration involves at least four major steps: reversible rolling, triggering by activation of integrins, stable adhesion, and diapedesis across the endothelium monolayer (1-3). Diapedesis is the final step in the transmigration process, during which neutrophils must change shape dramatically to pass through the endothelium monolayer. After transmigration, the neutrophils also continue migrating through the extracellular matrix (ECM), a three-dimensional (3-D) fiber mesh, until they reach the damaged or inflamed tissue (4). Mimicking such 3-D migration conditions in vitro is problematic because migration assays, such as those using a Boyden chamber, do not provide an appropriate 3-D microenvironment $(5,6)$.

Cell migration assays using hydrogels, networks of entangled polymer chains, are an alternative to 2-D migration assays.
A hydrogel-coated Boyden chamber membrane has been used to study migration (7-10), and hydrogel-based microfluidic devices also have been developed (11-14). Microfluidic devices can generate stable and controllable chemokine gradients by taking advantage of the diffusional mixing of laminar flow. Another advantage is that they can apply shear stress to cells, increasing their physiological relevance. Although these methods are promising and have produced interesting results, hydrogels and microfluidic devices are difficult to work with.

Nanofibrous membranes have a structure similar to the ECM of human tissue (15-18),

\section{METHOD SUMMARY}

Here we describe the use of electrospun nanofibrous membranes to study vertical migration of neutrophils. Neutrophils are first seeded and incubated on the surface of the nanofibrous membrane and then chemotactic responses are induced via addition of IL-8 blended agarose gel on the opposite side of the seeded surface. Samples can be kept in either the initial vertical arrangement, or flipped upside-down to change the direction of the gravitational force. 


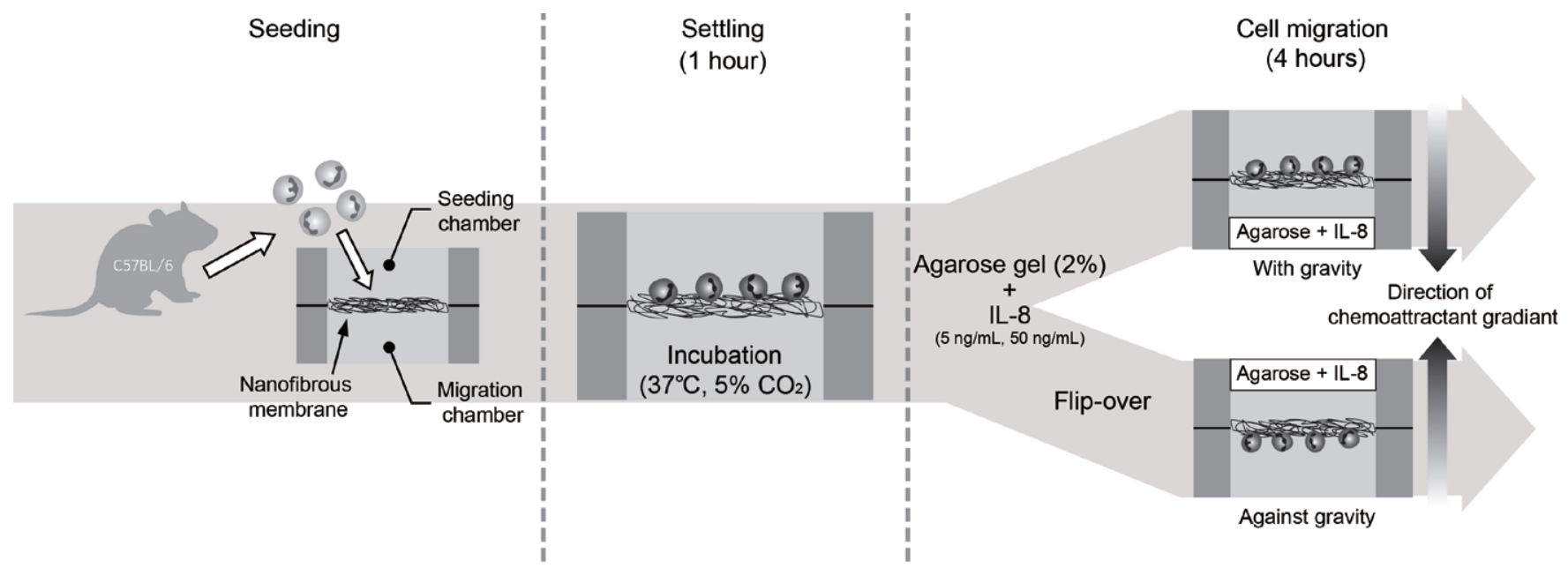

Figure 1. Experimental procedure for studying neutrophil migration through a nanofibrous membrane.

making them potentially valuable for studies of 3-D cell migration. Electrospinning is one of the most effective methods for manufacturing nanofibrous membranes; however, controlling the morphology and geometry of electrospun membranes and patterns remains a major challenge $(19,20)$. Recently, we presented a direct-write electrospinning (DWES) process and apparatus with improved focusing and scanning functionalities for generating various nanofibrous membranes with high geometric fidelity (19-22). As a result, we were able to fabricate nanofibrous membranes with uniform thicknesses of up to $150 \mu \mathrm{m}$ (20). The fabrication of such nanofibrous membranes with uniform thickness and structure is important for reproducibility of cell migration experiments.

Here we describe a nanofibrous membrane, fabricated using the DWES technique, for observing the 3-D migration of neutrophils. We demonstrated chemotactic migration against gravity, which is not observed in conventional migration assays. The migration assay consists of vertically arranged compartments separated by a nanofibrous membrane. Two migration conditions were tested: one was with gravity, in which the chemotactic gradient and the gravitational force have the same direction; and the other was against gravity, in which the chemotactic gradient and gravitational force have opposite directions. We demonstrated the 3-D vertical migration of neutrophils, including migration against gravity through the nanofibrous membrane. These results suggest that the nanofibrous membrane can substitute for the conventional membrane of the Boyden chamber to better mimic the 3-D in vivo migration environment.

\section{Materials and methods}

Fabrication of electrospun nanofibrous membranes

A nanofibrous membrane with uniform thickness and nanofiber density was fabricated using a direct-write electrospinning (DWES) method, which we reported previously $(19,20)$. The polymer for electrospinning was polycaprolactone ( $P C L$ ) with a numberaverage molecular weight of 80,000 (440744, Sigma-Aldrich, St Louis, MO), dissolved in chloroform (C0584, 99.5\%, Samchun Pure Chemical Co., Ltd., Seoul, South Korea) at a concentration of $8.8 \%$ by weight. During electrospinning, the temperature and relative humidity were $20^{\circ}-21^{\circ} \mathrm{C}$ and $50 \%-55 \%$, respectively, and the flow rate of the solution, voltage, and tip-to-collector-distance (TCD) were maintained at $0.1 \mathrm{~mL} /$ hour, $24-25 \mathrm{kV}$, and $65 \mathrm{~mm}$, respectively. With the DWES method, the fiber jet flowed directly from the nozzle to the collector. To fabricate the large electrospun nanofibrous membrane, the collector was translated through $X$ and $Y$, as well as axially (i.e., in the direction perpendicular to the plane), using the nozzle shaft. Nanofibrous membrane samples were composed of 30 sublayers, which were electrospun iteratively at a scanning speed of $30 \mathrm{~mm} / \mathrm{s}$ along a raster scan path with a pitch between scan lines of $125 \mu \mathrm{m}$. The fabricated nanofibrous membrane samples were $70 \pm$ 10- $\mu$ m-thick. The manufactured membranes were placed in a fume hood at room temperature for $24 \mathrm{~h}$, followed by baking in an oven at $60^{\circ} \mathrm{C}$ for $10 \mathrm{~s}$ to crosslink the fibers and evaporate the remaining chloroform. The membranes were soaked in $70 \%$ ethanol for $12 \mathrm{~h}$ for sterilization, washed 3 times using
PBS, and finally washed with fresh culture medium to minimize cytotoxicity due to any remaining ethanol or chloroform.

\section{Neutrophil isolation}

For the isolation of peritoneal neutrophils, mice were injected intraperitoneally with 3 $\mathrm{mL} 3 \%$ thioglycollate broth to recruit neutrophils (23). Six hours later, primary neutrophils were collected from euthanized animals by peritoneal lavage using $10 \mathrm{~mL}$ ice-cold EDTAbalanced salt solution (BSS) buffer. Cells were washed using ice-cold EDTA-BSS buffer. Red blood cells were lysed with RBC lysis buffer. Positive isolation of neutrophils was achieved with anti-Ly6G and Ly6C magnetic particles (MACS, Miltenyi, Bergisch Gladbach, Germany) in combination with the related cell separation system from Milteny iBiotec, following the manufacturer's protocol.

\section{Migration assays}

The migration assay chamber consisted of two vertically arranged compartments filled with HyClone RPMI 1640 medium (GE Healthcare Life Sciences, Logan, UT) supplemented with 10\% HyClone fetal bovine serum (FBS) (GE Healthcare Life Sciences) and the electrospun nanofibrous membrane. The nanofibrous membrane was sandwiched between the two compartments. We named the upper compartment the "seeding chamber" and the lower compartment the "migration chamber." Thus, the top surface of the nanofibrous membrane was the floor of the seeding chamber, and the bottom surface was the ceiling of the migration chamber. The chambers were composed of polydimethylsiloxane (PDMS) (Dow Corning, Midland, $\mathrm{MI}$ ) and bonded via corona treatment (24). 
Both chambers were sealed after seeding the neutrophils to prevent leakage of culture medium. Freshly harvested neutrophils (7.5 $\times 10^{4}$ cells) were seeded in the seeding chamber initially and incubated $1 \mathrm{~h}$ in an incubator $\left(37^{\circ} \mathrm{C}, 5 \% \mathrm{CO}_{2}\right)$ for settling. After settling, $5 \mathrm{ng} / \mathrm{mL}$ and $50 \mathrm{ng} / \mathrm{mL}$ of Interleukin 8 (IL-8) (PeproTech, Rocky Hill, NJ) was added to the migration chamber to induce a chemotactic response in the neutrophils $(25,26)$. We added an IL-8 blended agarose gel block ( $2 \%$ in distilled water) instead of adding IL-8 solution directly to prevent rapid diffusion of IL-8. The diffusion of IL-8 was estimated by measuring the intensity of $10-\mathrm{kDa}$ FITCdextran (Sigma Aldrich, St Louis, MO), which has a similar molecular weight to IL-8 (8.4 $\mathrm{kDa}$ ). The fluorescence intensity increased constantly over more than $12 \mathrm{~h}$, and we expected that the gradient of chemoattractant and the chemotactic response of the neutrophils would be maintained over a $12 \mathrm{~h}$ period (see Supplementary Figure S1).

We examined two migration conditions in terms of the direction of the gravitational force: with gravity and against gravity (Figure 1). For experiments with gravity, both the seeding and migration chambers were kept in the initial vertical arrangement with the direction of the gravitational force the same as the direction of migration. For experiments against gravity, samples were flipped upside down from the initial vertical orientation after settling so that the direction of the gravitational force was opposite the direction of migration. The Boyden chamber assay with 3 $\mu \mathrm{m}$ pores was used in the same experiments for comparison. Neutrophils migrated for $4 \mathrm{~h}$, and experiments were performed in triplicate.

\section{Results and discussion}

\section{Characteristics of fabricated} nanofibrous membranes

Figure 2A shows field-emission scanning electron microscope (FE-SEM) (Nova Nano 200; FEl Co., Hillsboro, OR) images of a fabricated nanofibrous membrane. The membranes had an almost flat, uniform surface, although fibers with varying diameters were electrospun. We also investigated the distribution of fiber diameter (Figure 2B), finding that most of the fibers had diameters of $200-500 \mathrm{~nm}$. In detail, the fiber diameter at peak frequency was $\sim 350 \mathrm{~nm}$, while the maximum diameter was $2000 \mathrm{~nm}$. Cell migration is influenced by the pore size of a membrane, so we investigated the pore size using a mercury porosimeter (AutoPore IV 9500; Micromeritics Instrument Co, Norcross, GA) with a penetrometer (s/n-14, 3 bulb, 0.412 stem, powder). The pressure for mercury filling was 1.23 psi, and the equilibrium time was 10 s. Figure $2 \mathrm{C}$ shows the measured distribution of the pore size (i.e., equivalent diameter) of the nanofibrous membrane. The membrane had varying pore sizes of up to several tens of micrometers, with the dominant pore size diameter of the membrane being between 3 and $4 \mu \mathrm{m}$. In addition, pores with diameters of 1-10 $\mu \mathrm{m}$ comprised approximately $77 \%$ of the total area.

PCL is known to exhibit good cell adhesion, and electrospun PCL membranes have greater adhesion ability when compared with smooth surfaces (27). In addition, adhesion of neutrophils on electrospun nanofibrous membranes was observed with or without serum in the culture media, but the neutrophils cultured with serum-containing medium showed better adhesion than those cultured with serum-free medium (Supplementary Figure S2).

\section{Neutrophil migration}

Migration of neutrophils through the membrane was evaluated by counting the number of neutrophils in each chamber. For all experimental conditions, neutrophils in the medium were collected from each chamber after $4 \mathrm{~h}$ of migration and were counted by fluorescence-activated cell sorting (FACS) by a Japan-made sorter, analyzer (JSAN) (Bay Bioscience, Kobe, Japan). We gently pipetted up and down 3-4 times before collecting media from each chamber to collect floating neutrophils. Figure 3A shows the migration of neutrophils tested using a Boyden chamber assay; the pore size was $3 \mu \mathrm{m}$, and the pore
A

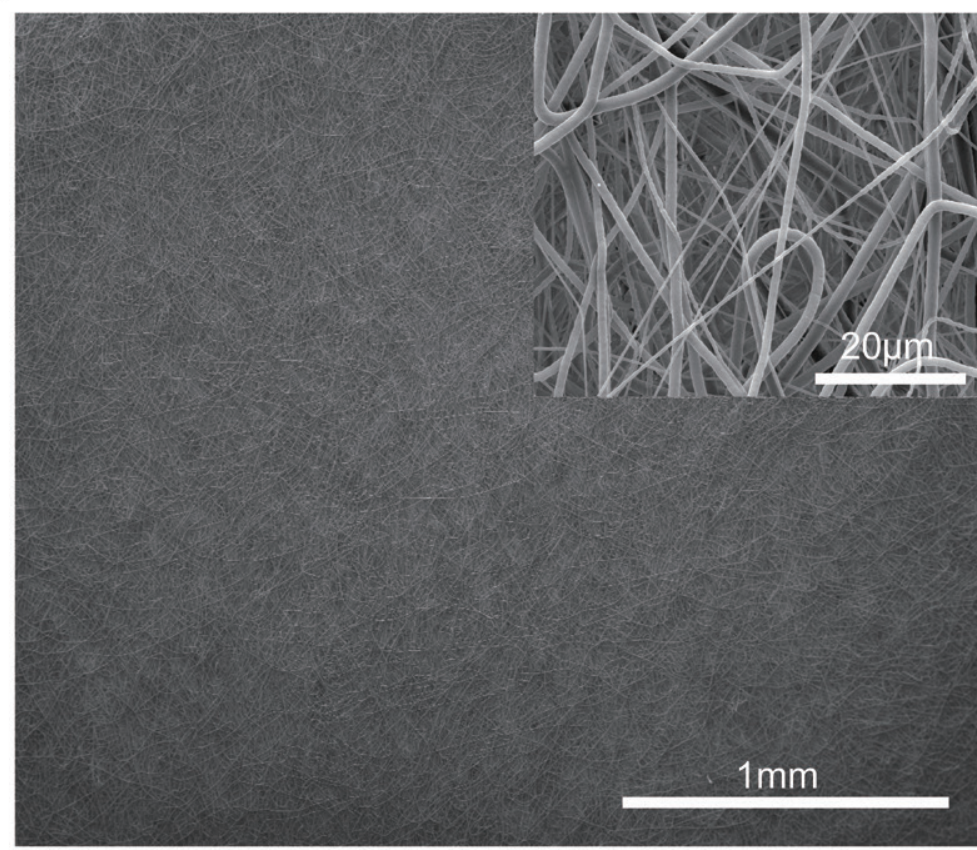

B

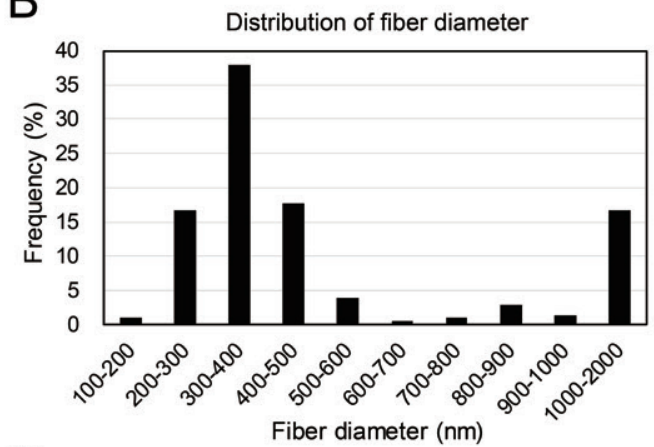

C

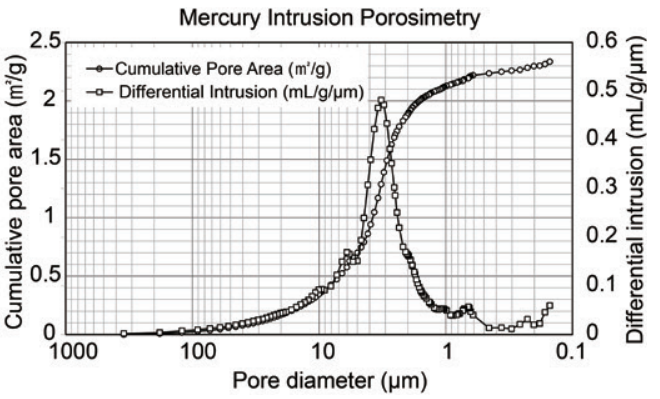

Figure 2. Structural properties of an electrospun nanofibrous membrane. (A) Scanning electron microscope (SEM) images of the fabricated nanofibrous membrane. (B) The fiber diameter distribution and (C) the equivalent pore size distribution and differential intrusion. The thickness of the membrane was $70 \pm 10 \mu m$. 
A
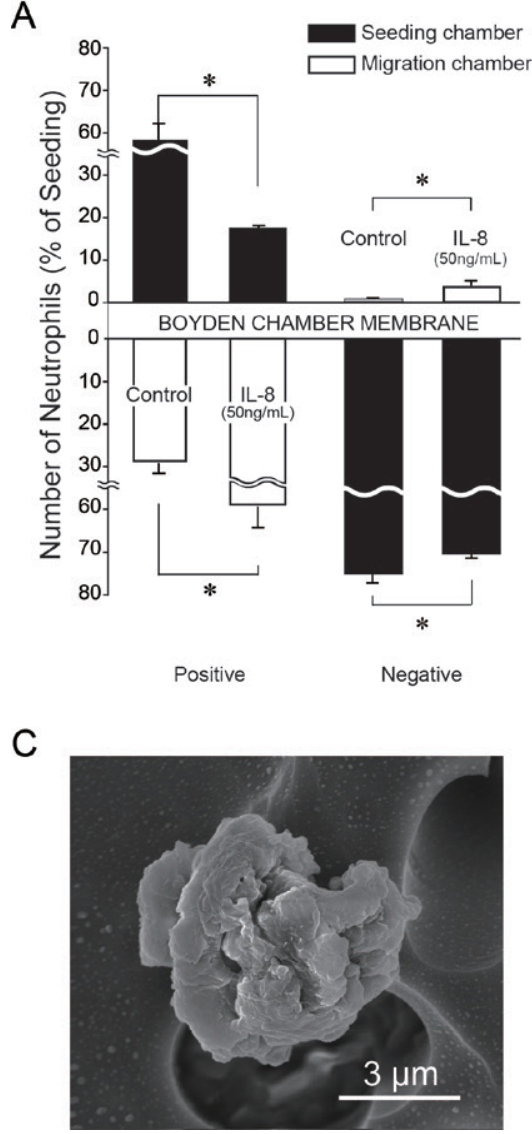

B

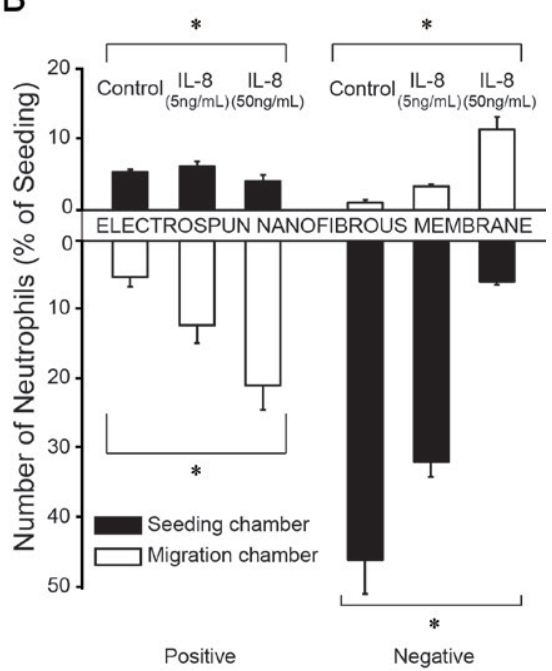

D

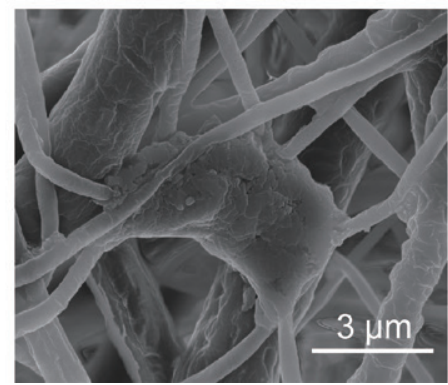

Figure 3. Migration of neutrophils measured using a Boyden chamber assay and a nanofibrous membrane assay. ( $A$ and $B$ ) Numbers of neutrophils in chambers containing either a conventional Boyden chamber membrane with 3- $\mu$ m pores (A) or a nanofibrous membrane (B). The error bars in the figures indicate the standard deviation $\left({ }^{*} P<0.04\right)$. Note that sum of the numbers of neutrophils in the seeding and migration chamber was $<100 \%$. This was due to neutrophils becoming stuck between the membrane nanofibers. (C and D) Scanning electron microscope (SEM) images of neutrophils on a conventional Boyden chamber membrane with 3- $\mu$ m pores (C) or a nanofibrous membrane (D).

density was $2 \times 10^{6}$ pores $/ \mathrm{cm}^{2}$. In experiments with gravity and a conventional Boyden chamber membrane, the chemotactic effect of IL-8 resulted in $60 \%$ of total neutrophils migrating from the seeding chamber to the migration chamber. Indeed, even in the absence of chemotactic stimulation, $~ 30 \%$ of total neutrophils migrated to the migration chamber. However, it was unclear whether the neutrophils migrated or simply fell through the pores due to gravity. In addition, this phenomenon was more intense for membranes with larger pores (i.e., 8 um) (Supplementary Figure S3). In experiments against gravity, most of the seeding neutrophils (>70\%) were found in the seeding chamber; fewer than 5\% migrated to the migration chamber, even in the presence of IL-8. Thus, the attachment force of neutrophils on the conventional membrane was not sufficient to withstand gravity, and the neutrophils simply fell off the membrane. The results of migration experiments using conventional membranes could be due to a mix of chemotactic migration and falling due to gravity; isolating the true extent of chemotactic migration from these results is problematic.

Figure $3 \mathrm{C}$ shows an FE-SEM photograph of neutrophils on a conventional membrane of a Boyden chamber. The surface of the conventional membrane, including the sidewalls of pores, is very smooth, and there are no anchors that could be used by a cell to migrate against gravity. This could be more important for cells that have amoeboid motility, such as neutrophils, because adhesive forces in amoeboid migration are lower than those of mesenchymal migration (28). In contrast, the nanofibrous membrane has a 3-D random structure consisting of entangled nanofibers; neutrophils cultured on the nanofibrous membrane attach along the nanofibers, as shown in Figure 3-D. Therefore only $\sim 5 \%$ of total neutrophils were found to have migrated in the control experiment; most got stuck between the nanofibers (Figure 3B). In the presence of $5 \mathrm{ng} / \mathrm{mL}$ and $50 \mathrm{ng} / \mathrm{mL}$ of $\mathrm{LL}-8,12 \pm 2.6 \%$ and $20.8 \pm 3.5 \%$ of the neutrophils migrated across the nanofibrous membrane to the migration chamber, respectively. Although the percentage of migrated neutrophils was much lower than seen with a conventional membrane, this result can be considered evidence of chemotactic migration through the nanofibrous membrane. In the case of experiments against gravity without IL-8, more neutrophils ( $45 \%)$ were found in the seeding chamber, and fewer than $2 \%$ migrated to the migration chamber. The ratio of migrated neutrophils increased to only $3.5 \%$ with 5 $\mathrm{ng} / \mathrm{mL}$ of IL-8; however, this ratio increased to $15 \%$ with $50 \mathrm{ng} / \mathrm{mL}$ of IL-8. It has been shown that the activation rate of neutrophils with a concentration of $50 \mathrm{ng} / \mathrm{mL}$ of IL-8 is approximately twice that of $5 \mathrm{ng} / \mathrm{mL}$ of $\mathrm{IL}-8$ (29), which explains the increased migration of neutrophils.

\section{Neutrophils inside the} nanofibrous membrane

We examined the distribution of neutrophils inside the nanofibrous membrane by taking cryosections (Figure 4). Prior to cryosectioning, the nanofibrous membrane collected from the migration assay chambers was washed twice with PBS and stained with 4,6-diamidino2-phenylindole (DAPI) (Invitrogen, Carlsbad, CA) to locate the neutrophils. The cryosection thickness was $12 \mu \mathrm{m}$, and 100 sections were made per membrane.

For all experimental conditions, the largest proportion of neutrophils was found at a shallow depth $(0-10 \mu \mathrm{m})$ beneath the seeding surface. Especially in the case of experiments with gravity, $60 \%$ (100 cells) of the neutrophils were found $0-10 \mu \mathrm{m}$ from the seeding surface. However, in the presence of IL-8, many of the neutrophils migrated toward the migration chamber, and the number of neutrophils at depths of 0-20 $\mu \mathrm{m}$ significantly decreased. For the control sample against gravity, only $\sim 45 \%$ of neutrophils were found near the seeding surface $(0-10 \mu \mathrm{m})$, and the number of neutrophils found in that band was much smaller (33 cells) than that of the control with gravity (100 cells). This indicates that although neutrophil attachment to the nanofibrous membrane was higher than to 
a conventional membrane, some of the neutrophils dropped down due to gravity. In the presence of IL-8, many cells in experiments with gravity migrated toward the IL-8-containing chamber; these findings agree with the cell counting results.

The neutrophils cultured on the nanofibrous membrane were analyzed with confocal microscopy (FV1200; Olympus, Tokyo, Japan) to compare the effect of IL-8 on their morphological characteristics. Cells were fixed and stained with DAPI, carboxyfluorescein diacetate succinimidyl ester (CFSE) (Invitrogen, Carlsbad, CA), and a monoclonal anti- $\beta$-actin antibody (mouse IgG2a isotype; Sigma Aldrich, St Louis, MO) to show the nucleus, membrane, and actin filaments, respectively. Images were taken after $4 \mathrm{~h}$ of incubation with IL-8 using the same assay as previously described. Figure 4C shows that the neutrophils exposed to IL-8 displayed a more irregular shape with protrusions (white arrows). In addition to the morphological changes, the distribution of actin (in red) showed marked changes. Actin was distributed peripherally in unstimulated control cells (see Figure 4C); however, IL-8 induced polarization of the neutrophils, as well as localized accumulation of actin at lamellipodia in the cells (see Figure 4D). Figure 4E shows the proportion of polarized neutrophils for the control and with $50 \mathrm{ng} / \mathrm{mL}$ of IL-8. Three samples were used for each set of conditions, and the samples were fixed and stained using DAPI and F-actin. We acquired nine images per sample at random positions, and the number of polarized neutrophils was counted manually (see Supplementary Figure S4). As shown in Figure 4E, $70 \%$ of the neutrophils were polarized with in the presence of $50 \mathrm{ng} / \mathrm{mL}$ IL-8, whereas only $21 \%$ were polarized in the absence of IL-8.

In conclusion, we have demonstrated 3-D chemotactic migration of neutrophils using an electrospun nanofibrous membrane. The nanofibrous membrane consists of randomly entangled nanofibers and provides a 3-D microenvironment similar to the ECM. We confirmed that neutrophils are able to migrate against gravity through a nanofibrous membrane, even though many of the seeded neutrophils were captured
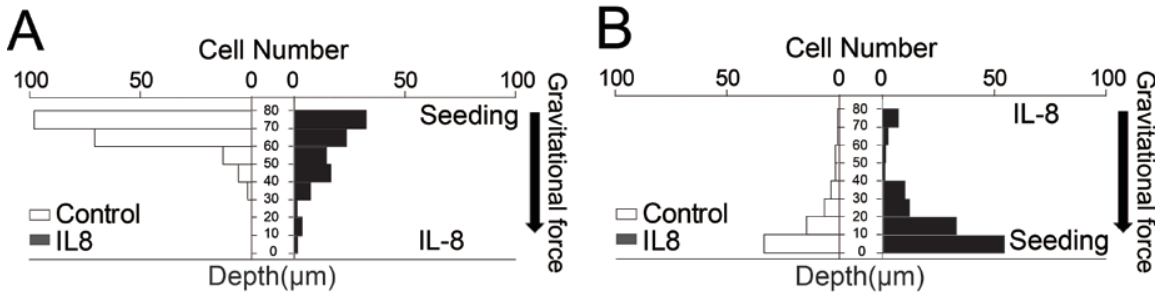

\section{C}
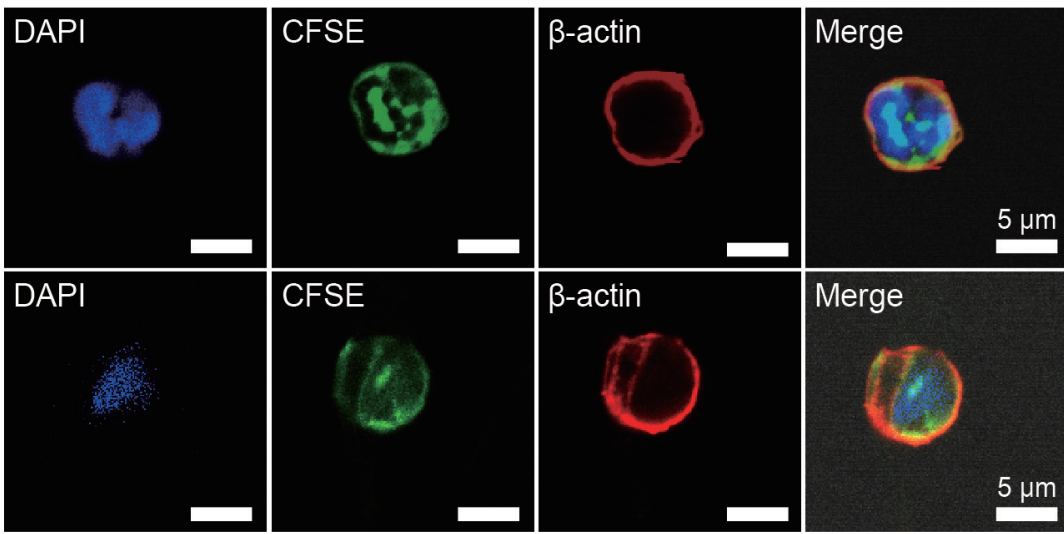

$D$
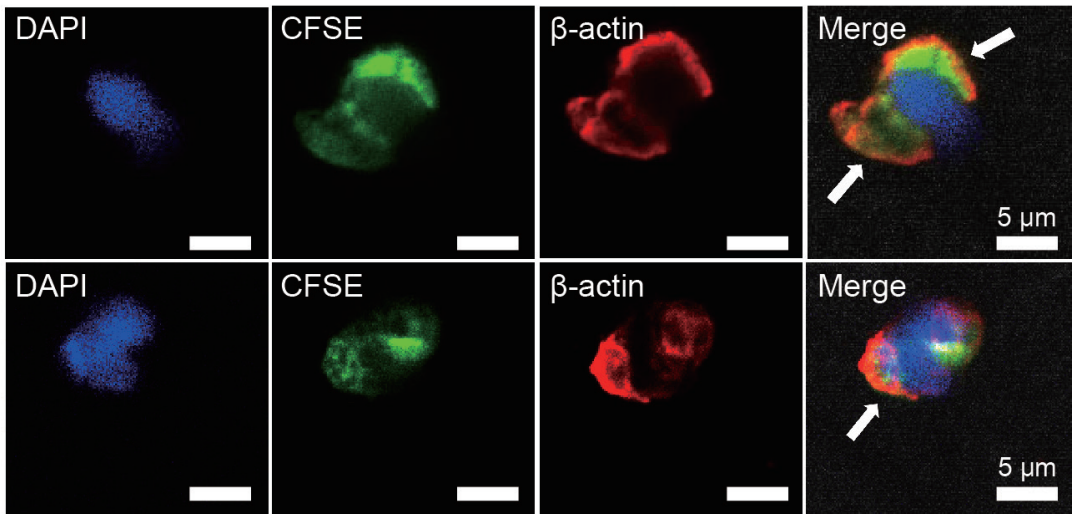

E

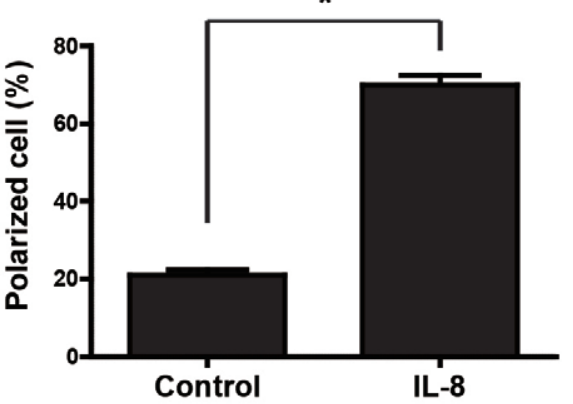

Figure 4. Neutrophils inside the nanofibrous membranes. (A) Distribution of neutrophils with gravity. (B) Distribution of neutrophils against gravity. (C and D) Confocal microscopy images of neutrophil morphology in a nanofibrous membrane under control conditions (C) or in the presence of $50 \mathrm{ng} / \mathrm{mL} \mathrm{IL}-8$ (D). White arrows indicate lamellipodia. (E) Proportion of polarized neutrophils under control conditions or in the presence of $50 \mathrm{ng} / \mathrm{mL}\left({ }^{\star} P<0.04\right)$.

inside of the membrane. Actin localization and morphological changes in the neutrophils stimulated by IL-8 were visualized using confocal microscopy. Natural directions for future research include using the assay to study the migration of various cell types and the effects of different types of cell-cell interactions.

\section{Author contributions}

All authors listed contributed significantly to the project. T.-M.P., B.D.L., and S.J. were responsible for all experiments. J.-S.K. and Y.H.J. supplied the nanofibrous materials. C.-H.K. and J.-Y.K. contributed biological techniques and analyzed data. S.Y. helped to separate and quantify the polarized cells. 


\section{Acknowledgments}

This research was supported by the Pioneer Research Center Program through the National Research Foundation of Korea funded by the Ministry of Science, ICT \& Future Planning (NRF-2012-0009666).

\section{Competing interests}

The authors declare no competing interests.

\section{References}

1. Johnson-Léger, C., M. Aurrand-Lions, and B.A. Imhof. 2000. The parting of the endothelium: miracle, or simply a junctional affair? J. Cell Sci. 113:921-933.

2. Luster, A.D., R. Alon, and U.H.v. Andrian. 2005. Immune cell migration in inflammation: present and future therapeutic targets. Nat. Immunol. 6:11821190

3. Beltman, J.B., A.F.M. Marée, and R.J.d. Boer. 2009. Analysing immune cell migration. Nat. Rev. Immunol. 9:789-798.

4. Ley, K. 1996. Molecular mechanisms of leukocyte recruitment in the inflammatory process. Cardiovasc. Res. 32:733-742.

5. Gerthoffer, W.T. 2007. Mechanisms of vascular smooth muscle cell migration. Circ. Res. 100:607-621.
6. Klamer, S.E., C.G. Kuijk, P.L. Hordijk, C.E. van der Schoot, M. von Lindern, P.B. van Hennik, and C. Voermans. 2013. BIGH3 modulates adhesion and migration of hematopoietic stem and progenitor cells. Cell Adh Migr. 7:434-449.

7. Toetsch, S., P. Olwell, A. Prina-Mello, and Y. Volkov. 2009. The evolution of chemotaxis assays from static models to physiologically relevant platforms. Integr Biol (Camb). 1:170-181.

8. Agren, M.S. 1998. An amorphous hydrogel enhances epithelialisation of wounds. Acta Derm Venereol. 78:119-122.

9. Bock, O. and U. Mrowietz. 2001. Development of a new method of analysing chemotactic deactivation of human neutrophil granulocytes. J. Biochem. Biophys. Methods 48:257-268.

10. Bloomfield, K.L., B.L. Baldwin, D.G. Harkin, and K.F. Tonissen. 2001. Modification of the Boyden chamber to improve uniformity of cell invasion of matrigel coated membranes. Biotechniques 31:12421246.

11. Cheng, S.-Y., S. Heilman, M. Wasserman, S. Archer, M.L. Shulerac, and M. Wua. 2007. A hydrogel-based microfluidic device for the studies of directed cell migration. Lab Chip. 7:763-769.

12. Bettinger, C.J. and J.T. Borenstein. 2010. Biomaterials-based microfluidics for engineered tissue constructs. Soft Matter 6:4999-5015.

13. Kim, D. and D.J. Beebe. 2007. Hydrogel-based reconfigurable components for microfluidic devices. Lab Chip. 7:193-198.

\section{In vivo grade isotype control antibodies}

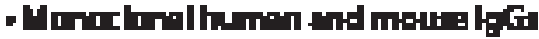

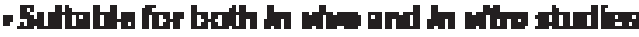

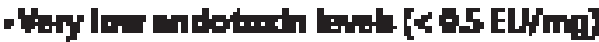 \\ - Supplied at hlah monartmon (2-5 mg/mi)

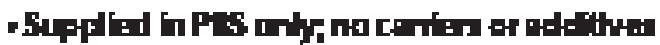

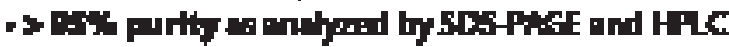

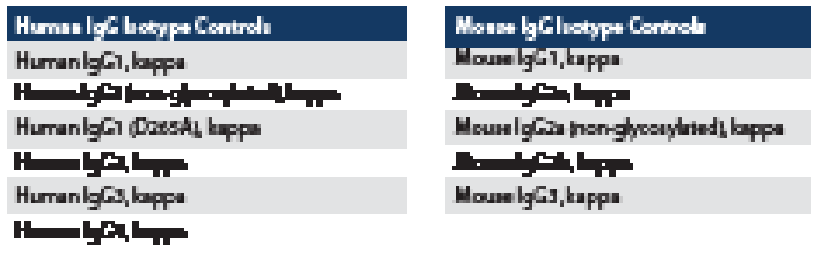

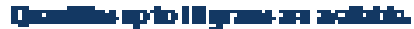

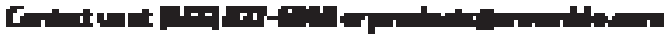

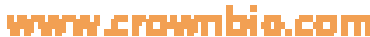

14. Wan, J. 2012. Microfluidic-Based Synthesis of Hydrogel Particles for Cell Microencapsulation and Cell-Based Drug Delivery. Polymers 4:1084-1108.

15. Tambralli, A., B. Blakeney, J. Anderson, M. Kushwaha, A. Andukuri, D. Dean, and H.-W. Jun. 2009. A hybrid biomimetic scaffold composed of electrospun polycaprolactone nanofibers and selfassembled peptide amphiphile nanofibers. Biofabrication 1:025001.

16. Kolambkar, Y.M., K.M. Dupont, J.D. Boerckel, N. Huebsch, D.J. Mooney, D.W. Hutmacher, and R.E. Guldberg. 2011. An alginate-based hybrid system for growth factor delivery in the functional repair of large bone defects. Biomaterials 32:65-74.

17. Xie, Z., C.B. Paras, H. Weng, P. Punnakitikashem, L.-C. Su, K. Vu, L. Tang, J. Yang, and K.T. Nguyen. Dual growth factor releasing multifunctional nanofibers for wound healing. Acta Biomater. (In press).

18. Pham, Q.P., U. Sharma, and A.G. Mikos. 2006. Electrospinning of polymeric nanofibers for tissue engineering applications: a review. Tissue Eng. 12:1197-1211.

19. Lee, J., S.Y. Lee, J. Jang, Y.H. Jeong, and D.-W. Cho. 2012. Fabrication of patterned nanofibrous mats using direct-write electrospinning. Langmuir 28:7267-7275.

20. Lee, J., Y.H. Jeong, and D.-W. Cho. 2014. Fabrication of nanofibrous mats with uniform thickness and fiber density. Macromol. Mater. Eng. 299:10521061

21. Lee, J., J. Jang, H. Oh, Y.H. Jeong, and D.-W. Cho. 2013. Fabrication of a three-dimensional nanofibrous scaffold with lattice pores using directwrite electrospinning. Mater. Lett. 93:397-400.

22. Lee, S.Y., B.R. Lee, J. Lee, S. Kim, J.K. Kim, Y.H. Jeong, and S. Jin. 2013. Microscale Diffusion Measurements and Simulation of a Scaffold with a Permeable Strut. Int. J. Mol. Sci. 14:20157-20170.

23. Lagasse, E. and I.L. Weissman. 1994. bcl-2 Inhibits Apoptosis of Neutrophils but Not Their Engulfment by Macrophages. J. Exp. Med. 179:1047-1052.

24. Goldman, M., A. Goldman, and R.S. Sigmond. 1985. The corona discharge, its properties and specific uses. Pure Appl. Chem. 57:1353-1362.

25. Smith, W.B., J.R. Gamble, I. Clark-Lewis, and M.A. Vadas. 1991. Interleukin-8 induces neutrophil transendothelial migration. Immunology 72:65-72.

26. Nolan, S., R. Dixon, K. Norman, P. Hellewell, and V. Ridger. 2008. Nitric oxide regulates neutrophi migration through microparticle formation. Am. J. Pathol. 172:265-273.

27. Ruckh, T.T., K. Kumar, M.J. Kipper, and K.C Popat. 2010. Osteogenic differentiation of bone marrow stromal cells on poly(e-caprolactone) nanofiber scaffolds. Acta Biomater. 6:2949-2959.

28. Friedl, P. and K. Wolf. 2003. Tumour-cell invasion and migration: diversity and escape mechnisms. Nat. Rev. Cancer 3:362-374.

29. Sato, E., K.L. Simpson, M.B. Grisham, S Koyama, and R.A. Robbins. 2000. Reactive Nitrogen and Oxygen Species Attenuate Interleukin8-induced Neutrophil Chemotactic Activity in Vitro. J. Biol. Chem. 275:10826-10830.

Received 14 August 2014; accepted 12 March 2015.

Address correspondence to Songwan Jin, Department of Mechanical Engineering, Korea Polytechnic University, Siheung, Gyeonggi-do, South Korea. E-mail: songwan@ kpu.ac.kr

To purchase reprints of this article, contact: biotechniques@fosterprinting.com 